\title{
High repetition rate, high pulse energy, Raman shifted wavelength selectable fiber laser source in the visible
}

\author{
L. Xu, S. Alam, Q. Kang, D.P. Shepherd and D.J. Richardson \\ Optoelectronics Research Centre, University of Southampton, Southampton, SO17 1BJ, UK \\ l.xu@soton.ac.uk
}

\begin{abstract}
We have demonstrated a Raman-shifted wavelength-selectable fiber laser source with fundamental spatial-mode output producing $\sim 1-\mu \mathrm{J}$ pulse-energy at 1-MHz repetition-rate with 1.3ns pulse-width using a large-core photonic crystal fiber.

OCIS codes: (140.0140) Lasers and laser optics; (140.3550) Lasers, Raman
\end{abstract}

\section{Introduction}

Photoacoustic microscopy (PAM) is an emerging technology that provides high-contrast imaging based on physiological differences in the tissue optical absorption[1]. Optical-resolution photoacoustic microscopy (ORPAM) is capable of achieving optical-absorption-contrast imaging with micron-scale resolution[2]. For OR-PAM, pulsed lasers with $\mu \mathrm{J}$-level energies, good beam quality and excitation wavelengths in the visible and near-infrared are generally required. Compared to bulk laser and dye laser sources, fibre laser technology offers the prospect of compact, reliable and efficient photoacoustic excitation sources for clinical use. In addition, fibre lasers can provide high pulse repetition frequencies to achieve rapid image acquisition[3]. Cascaded stimulated Raman scattering (SRS) in optical fibres provides a convenient and practical mechanism to increase the number of excitation wavelengths for functional photoacoustic microscopy[4]. Selective excitation of Raman Stokes wavelengths can be realized by using a flat-topped rectangular pump pulse, since all points across the pulse experience identical Raman gain. Using this concept, selective excitation of individual Raman Stokes lines of up to the ninth order has been reported previously by our group[5]. However, it was found that with increased pump peak power, the suppression ratio of each excited Stokes line decreased and also the spectral bandwidths of the Stokes lines were broadened by other nonlinear effects. The resulting energy spectral density was then low due to the distribution of energy over a wide spectral range. Here, we report an investigation of selective generation of high-pulse-energy Raman Stokes lines by cascaded SRS in a number of different fibre geometries. By choosing large-mode-area (LMA) photonic crystal fibre (PCF), $\sim 1-\mu \mathrm{J}$ energy, 1-MHz repetition rate, 1.3-ns pulses with single-mode beam quality and relatively narrow spectral bandwidth have been achieved at the Raman Stokes wavelengths of $560 \mathrm{~nm}$ (2nd Stokes) and 575 $\mathrm{nm}$, (3rd Stokes), when pumped by a frequency-doubled Yb-doped-fibre master oscillator power amplifier (MOPA) system. These wavelengths are targeted as they are located in the absorption spectra of oxy and deoxy haemoglobin,

\section{Experiment and results}

As depicted in Fig. 1, the nanosecond MOPA consisted of a continuous wave (CW) polarization-maintaining (PM)fibre-pigtailed tunable diode laser as the seed, followed by a 5-stage Yb-doped fibre-amplifier chain. An electrooptic modulator (EOM) was used after the first pre-amplifier to chop the amplified CW radiation into a pulsed format with a 1-MHz repetition rate and 1.3-ns pulse duration. The first and second amplifiers were core-pumped (5$\mu \mathrm{m}$ core size), and the third and fourth amplifiers were cladding-pumped (10- $\mu \mathrm{m}$ core size). An acoustic-optic modulator (AOM) was inserted after the third amplifier in order to eliminate the amplified spontaneous emission (ASE) between pulses and also to increase the dynamic range of the amplified pulses. The final-stage power amplifier comprised a LMA Yb-doped fibre with a core diameter of $25 \mu \mathrm{m}$. The MOPA output wavelength (1064 $\mathrm{nm})$ was converted to the second-harmonic $(532 \mathrm{~nm})$ after passing through a 25 -mm-long LBO crystal with noncritical type-I phase-matching. The second harmonic generation (SHG) had a conversion efficiency of $60 \%$ and a maximum available pulse energy of $12 \mu \mathrm{J}$ and this was used to pump cascaded SRS in a number of different fibre geometries.

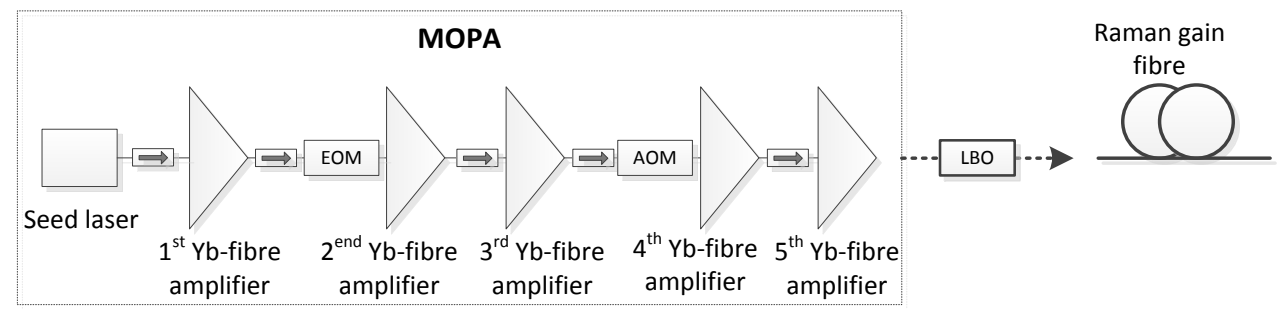

Fig. 1. Schematic of the experimental setup 
In the experiment, we first tested a step-index single-mode fibre (SMF) (S405-XP, Thorlabs), which had a pure silica core with a diameter of $\sim 4$ um and a cut-off wavelength of $380 \mathrm{~nm}$. By using a 15-meter length of SMF, the optimized selectively excited Raman Stokes spectral components had linewidths ( $3 \mathrm{~dB}$ ) of $2.6 \mathrm{~nm}$ and $3.3 \mathrm{~nm}$ for the 2nd and 3rd order Stokes, respectively. The calculated pulse energies constrained within the 3-dB bandwidth were $0.5 \mu \mathrm{J}$ for both Stokes lines. Any further increase in input power would have depleted the 3rd Stokes pulse and shifted the output to longer wavelengths not required for our particular application. In an attempt to achieve higher pulse energies at the 2nd and 3rd Stokes wavelengths, we shortened the fibre length and increased the input pulse energy. For a 5-meter-long SMF, the 2nd and 3rd Stokes spectra were broadened in comparison to that obtained with the longer fibre, as shown in Fig. 2 (b). The 3-dB bandwidth of the 2nd and 3rd Stokes spectra were increased to $5.7 \mathrm{~nm}$ and $12.3 \mathrm{~nm}$, respectively, containing pulse energies of $0.9 \mu \mathrm{J}$ and $1.0 \mu \mathrm{J}$, significantly reducing the spectral energy density. The SRS line broadening is thought to be due to self-phase modulation (SPM) due to the increased intensity. Since the peak non-linear phase shift of SPM has a linear dependency on fibre length, while the Raman gain has an exponential dependency, the use of a shorter fibre length and higher pulse energy has led to an increase in the relative impact of SPM on the output spectrum.

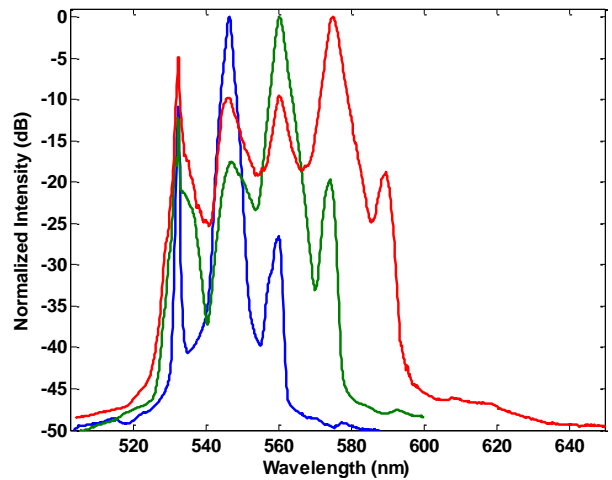

(a)

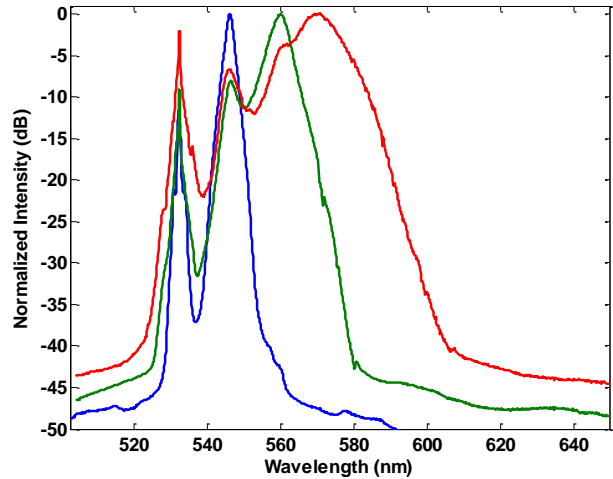

(b)

Fig. 2. Output Raman spectra from a step-index single mode fibre with lengths of $15 \mathrm{~m}$ (a) and $5 \mathrm{~m}$ (b). The different colours represent different pump powers to selective excite different Stokes lines: blue (1st Stokes); green (2nd Stokes); red (3rd Stokes).

Considering the length-scaling rules of SPM versus Raman scattering, an increase in the fibre core size rather than reduction in the fibre length was considered to be a more promising way to scale the Stokes pulse energy. We therefore used a large core $(\sim 17 \mu \mathrm{m})$ graded-index multi-mode fibre (GIMMF) as it has been shown that a gradedindex profile can lead to SRS beam clean-up in multi-mode fibres due to the lower-order transverse Stokes modes experiencing more gain than the higher-order transverse Stokes modes [6]. Hence, we hoped to be able to obtain fundamental-mode beam quality, while avoiding SPM-induced spectral broadening. We observed the clean-up effect in an experiment with a 1-km long GIMMF, where the Raman Stokes output beam was found to be in the fundamental mode despite the pump being highly multi-moded. A shorter length of $19 \mathrm{~m}$ was then used for selectively exciting Stokes lines up to 3rd order with high pulse energy. Due to larger core, the 3-dB bandwidths of the Stokes-shifted components in the GIMMF were indeed narrowed to $2.3 \mathrm{~nm}$ and $2.7 \mathrm{~nm}$ for the central wavelengths of $560 \mathrm{~nm}$ and $575 \mathrm{~nm}$. However, as shown in Fig. 3, the Stokes output beam was not in a clean fundamental mode, which we attribute to the short length of fibre not being sufficient for Raman clean-up effects to dominate.

Photonic crystal fibres (PCFs) can be designed with a large core surrounded by a cladding consisting of a periodic structure that can produce remarkable optical properties, such as endlessly single-mode operation. In order to simultaneously obtain diffraction limited beam quality, $\mu \mathrm{J}$ pulse energies, and relatively narrow linewidths for the Raman shifted output, we used an endlessly single-mode PCF, with a core diameter of $10 \mu \mathrm{m}$ (LMA-10, NKT). For a length of $19 \mathrm{~m}$, selectively excited narrow-spectral-bandwidth Raman Stokes beams, with fundamental beam quality, were successfully achieved. The 3-dB bandwidth of the 2nd and 3rd order Stokes spectral components were $2.9 \mathrm{~nm}$ and $3.5 \mathrm{~nm}$, respectively, and the corresponding pulse energies were $1.0 \mu \mathrm{J}$ and $0.9 \mu \mathrm{J}$. 


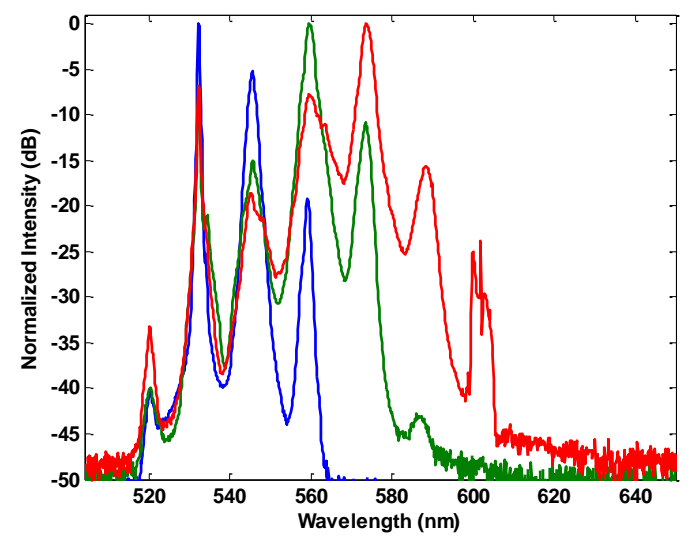

(a)

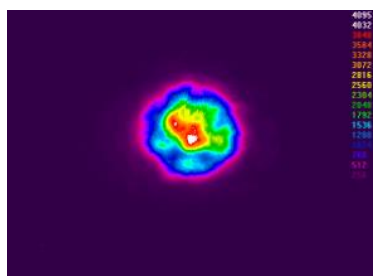

1st Stokes beam

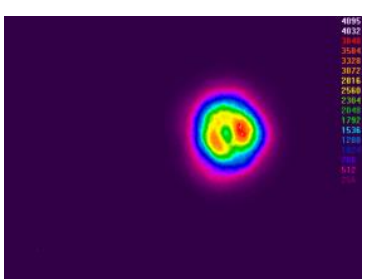

3rd Stokes beam

(b)

Fig. 3. (a) Output Raman spectra of graded-index fibre and (b) output beam profiles.

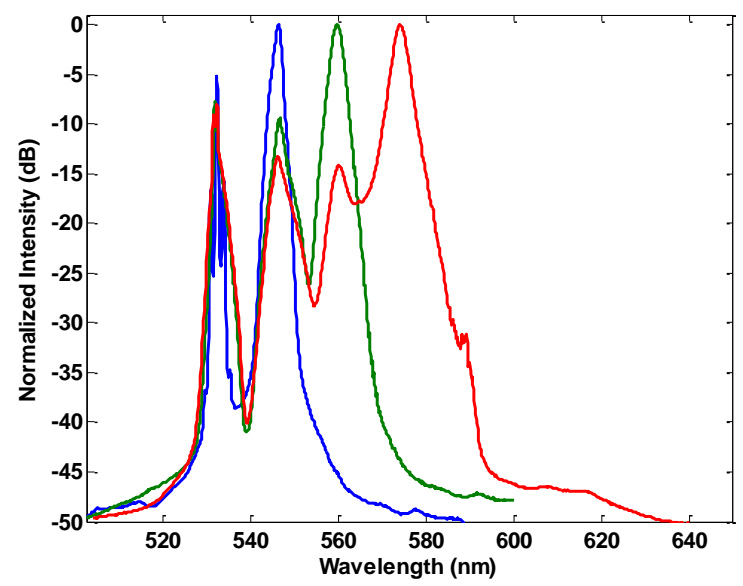

Fig. 4. Output Raman spectra of photonic crystal fibre.

\section{Conclusions}

In conclusion, we have investigated and demonstrated fibre laser sources of discrete Raman-shifted wavelengths with high pulse energy and good beam quality. We have shown that the length scaling laws of the Raman gain and SPM dictate the use of larger core fibres. We also show that Raman clean-up in graded-index multi-mode fibre is not strong enough to obtain diffraction limited beam quality in the short lengths of fibre required to obtain the high pulse energies required. By using a PCF, a wavelength-selective fibre laser source with diffraction limited output beam with $\sim 1-\mu \mathrm{J}$ pulse energy at $1-\mathrm{MHz}$ repetition rate was obtained. The final source has the desired parameters for OR-PAM applications with fast acquisition speed.

\section{References}

[1] J. Yao, L. Wang, J.-M. Yang, K. I. Maslov, T. T. W. Wong, L. Li, C.-H. Huang, J. Zou, and L. V. Wang, "High-speed label-free functional photoacoustic microscopy of mouse brain in action," Nature Methods 12, 407-410 (2015).

[2] K. Maslov, H. F. Zhang, S. Hu, and L. V. Wang, "Optical-resolution photoacoustic microscopy for in vivo imaging of single capillaries," Optics Letters 33, 929-931 (2008).

[3] W. Shi, P. Hajireza, P. Shao, A. Forbrich, and R. J. Zemp, "In vivo near-realtime volumetric optical-resolution photoacoustic microscopy using a high-repetition-rate nanosecond fiber-laser," Optics Express 19, 17143-17150 (2011).

[4] P. Hajireza, A. Forbrich, and R. Zemp, "In-Vivo functional optical-resolution photoacoustic microscopy with stimulated Raman scattering fiber-laser source," Biomed. Optics Express 5, 539-546 (2014).

[5] K. K. Chen, S.-u. Alam, P. Horak, C. A. Codemard, A. Malinowski, and D. J. Richardson, "Excitation of individual Raman Stokes lines in the visible regime using rectangular-shaped nanosecond optical pulses at $530 \mathrm{~nm}$," Optics Letters 35, 2433-2435 (2010).

[6] N. B. Terry, T. G. Alley, and T. H. Russell, "An explanation of SRS beam cleanup in graded-index fibers and the absence of SRS beam cleanup in step-index fibers," Optics Express 15, 17509-17519 (2007). 